\title{
Two-dimensional Hybrid Simulations of Kinetic Plasma Turbulence: Current and Vorticity vs Proton Temperature
}

\author{
Luca Franci ${ }^{1,2}$, Petr Hellinger ${ }^{3, a)}$, Lorenzo Matteini ${ }^{4}$, Andrea Verdini ${ }^{1,5}$ and Simone \\ Landi ${ }^{1,6}$ \\ ${ }^{1}$ Dipartimento di Fisica e Astronomia, Università degli Studi di Firenze, Largo E. Fermi 2, I-50125 Firenze, Italy \\ ${ }^{2}$ INFN-Sezione di Firenze, Via G. Sansone 1, I-50019 Sesto F.no (Firenze), Italy \\ ${ }^{3}$ Astronomical Institute, AS CR, Bocni II/1401, CZ-14100 Prague, Czech Republic \\ ${ }^{4}$ Physics Department, Imperial College London, London SW7 2AZ, UK \\ ${ }^{5}$ Solar-Terrestrial Center of Excellence, Royal Observatory of Belgium, Brussels, Belgium \\ ${ }^{6}$ INAF-Osservatorio Astrofisico di Arcetri, Largo E. Fermi 5, I-50125 Firenze, Italy \\ ${ }^{a)}$ Corresponding author: petr.hellinger@asu.cas.cz
}

\begin{abstract}
Proton temperature anisotropies between the directions parallel and perpendicular to the mean magnetic field are usually observed in the solar wind plasma. Here, we employ a high-resolution hybrid particle-in-cell simulation in order to investigate the relation between spatial properties of the proton temperature and the peaks in the current density and in the flow vorticity. Our results indicate that, although regions where the proton temperature is enhanced and temperature anisotropies are larger correspond approximately to regions where many thin current sheets form, no firm quantitative evidence supports the idea of a direct causality between the two phenomena. On the other hand, quite a clear correlation between the behavior of the proton temperature and the out-of-plane vorticity is obtained.
\end{abstract}

\section{INTRODUCTION}

The solar wind is a highly turbulent plasma. This idea is supported by the power-law behavior of its observed energy spectra, which span nearly four decades in frequency, from large to small kinetic scales (e.g., [1]). Among other things, in situ measurements also reveal the presence of an ubiquitous proton temperature anisotropy between the direction parallel and perpendicular to the mean magnetic field $[2,3]$. Vlasov-Hybrid simulations suggest that such temperature anisotropy and non-Maxwellian kinetic effects are mostly found around peaks of the current density [4, 5, 6, 7].

Recently, high-resolution two-dimensional (2D) hybrid particle-in-cell simulations have proved to be a reliable, state-of-the-art tool to investigate the properties of kinetic plasma turbulence, provided that a sufficiently large number of particles-per-cell is employed, especially when trying to quantitatively estimate the perpendicular proton temperature. In particular, the direct numerical simulations shown in $[8,9]$ have been able to recover simultaneously several features observed in the solar wind spectra, e.g.: i) a power-law behavior for the magnetic, kinetic and residual energy spectra with different spectral indices (e.g., [10]), ii) a magnetic spectrum with a smooth break at proton scales and a power-law scaling in the sub-proton range with a spectral index of $\sim-2.8$ (e.g., [11]), iii) an increase in magnetic compressibility at small scales (e.g., [12]), iv) a strong coupling between density and magnetic fluctuations in the kinetic range (e.g., [10]).

In the present paper, we show new complementary results coming from the 2D hybrid particle-in-cell simulations already presented in $[8,9]$. In particular, we will focus our attention on the correlations between the peaks in the outof-plane vorticity and the proton temperature enhancement and anisotropy. 


\section{RESULTS}

The numerical results discussed here were obtained by means of the hybrid particle-in-cell code CAMELIA, which treats electrons as a massless, charge neutralizing, isothermal fluid, whereas ions as particles. The characteristic spatial unit is the proton inertial length, $d_{p}$. We employ a 2D square computational grid in the $(x, y)$ plane, with periodic boundary conditions, $2048^{2}$ square cells and a total length $L=256 d_{p}$. Each cell has a size of $0.125 d_{p}$ and contains 8000 particles representing protons. The number density is assumed to be equal for protons and electrons and both species are isotropic, with the same plasma beta, $\beta=0.5$. The initial proton temperature anisotropy is set to $A_{p}=T_{p \perp} / T_{p \|}=1$, where $T_{p \perp}$ and $T_{p \|}$ are the perpendicular and parallel proton temperatures, respectively. We impose an initial ambient magnetic field $\boldsymbol{B}_{0}=B_{0} \hat{z}$, perpendicular to the simulation plane, and an initial spectrum of linearly polarized magnetic and bulk velocity fluctuations with only in-plane components. Fourier modes of equal amplitude and random phases are excited, assuring energy equipartition and vanishing correlation between kinetic and magnetic fluctuations. Fields are defined as parallel $(\|)$ and perpendicular $(\perp)$ with respect to the mean magnetic field, whereas the proton temperatures are intended with respect to the local magnetic field, $\boldsymbol{B}=\boldsymbol{B}{ }_{0}+\delta \boldsymbol{B}$, where $\delta \boldsymbol{B}$ are the fluctuations. For further information about the numerical setting and parameters and for a complete definition of all quantities, please refer to [8, 9].
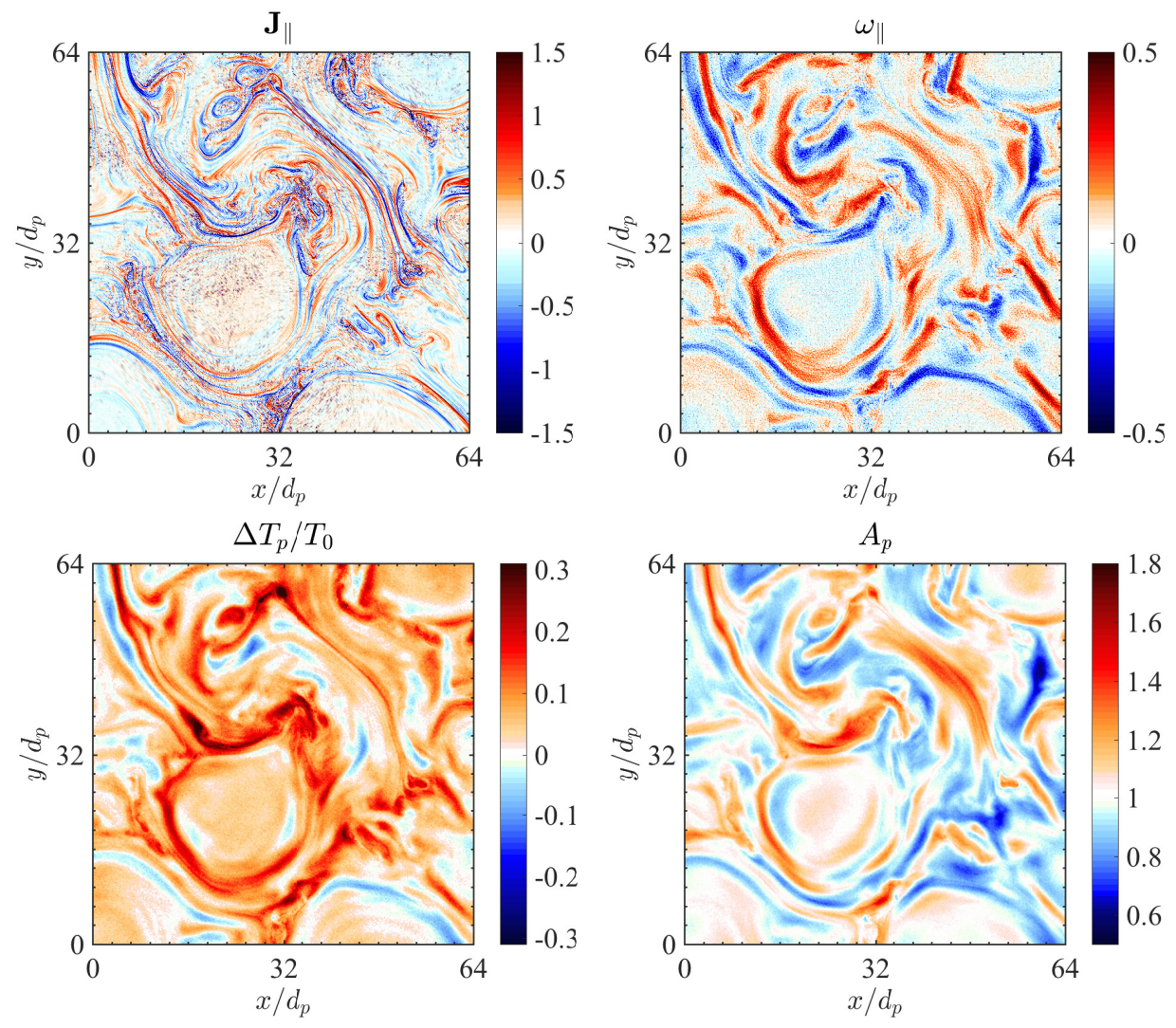

FIGURE 1. Contour plots of four different quantities on a portion of the $(x, y)$ plane, i.e., $\left[0,64 d_{p}\right] \times\left[0,64 d_{p}\right]$, at the time of maximum turbulent activity: the out-of-plane current density, $\boldsymbol{J}_{\|}$(top-left panel), the out-of-plane vorticity, $\boldsymbol{\omega}_{\|}$(top-right panel), the proton temperature variation normalized to the initial temperature, $\Delta T_{p} / T_{0}$ (bottom-left panel), and the proton temperature anisotropy, $A_{p}$ (bottom-right panel).

Figure 1 shows isocontours of four different quantities in a small portion of the $2 \mathrm{D}$ simulation domain, i.e., $\left[0,64 d_{p}\right] \times\left[0,64 d_{p}\right]$. All these snapshots have been taken at the time of maximum turbulent activity, which corresponds to the peak of the RMS value of the out-of-plane current density, $J_{\|}=(\nabla \times \boldsymbol{B})_{\|}$, during its time evolution [8, 9]. 
In the top-left panel, we show the local spatial distribution of the out-of-plane current density, $J_{\|}$. By the time a turbulence cascade has fully developed, many thin current sheets have already formed and partially disrupted, generating a complex pattern with small-scale structures. In the top-right panel, we report the out-of-plane vorticity, $\omega_{\|}=(\nabla \times \boldsymbol{u})_{\|}$, where $\boldsymbol{u}$ is the proton bulk velocity. It seems to follow a similar pattern as the one of $\boldsymbol{J}_{\|}$, although with a much less filamentary structure. Peaks of $\omega_{\|}$and peaks of $\boldsymbol{J}_{\|}$occupy approximately the same regions, although the latter exhibits a more structured pattern and it usually fills the spaces between the structures of the former. In the bottom-left panel, we report the normalized proton temperature variation, $\Delta T_{p} / T_{0}=\left(T_{p}-T_{0}\right) / T_{0}$, where $T_{p}=\left(2 T_{p \perp}+T_{p \|}\right) / 3$ is the average proton temperature at the time of maximum turbulent activity. Although $\Delta T_{p}$ can be locally both negative or positive, the resulting global proton temperature enhancement is clearly positive, and the same holds when the whole domain is considered (cf. [9]). Finally, the proton temperature anisotropy, $A_{p}$, is reported in the bottom-right panel. It ranges about between 0.6 and 1.6 in this portion of the computational domain (a similar range of values is reached in the whole box). This wide excursion is a signature of a strong local reshaping of particle distributions, leading to both perpendicular and parallel anisotropies [13].

If we now compare the local spatial distribution of these four quantities, we see that proton temperature enhancements and a quite strong proton temperature anisotropy seem to occur in the vicinity of current sheets (cf., [5]). Nevertheless, if we now focus on the structure of the out-of-plane vorticity, we realize that it matches the shapes of the two quantities related to the proton temperatures even better. Moreover, areas with a positive vorticity usually correspond to an increase in the total proton temperature and to the proton temperature anisotropy larger than $1\left(T_{p \perp}>T_{p \|}\right)$, whereas the opposite behavior occurs in areas with a negative vorticity.
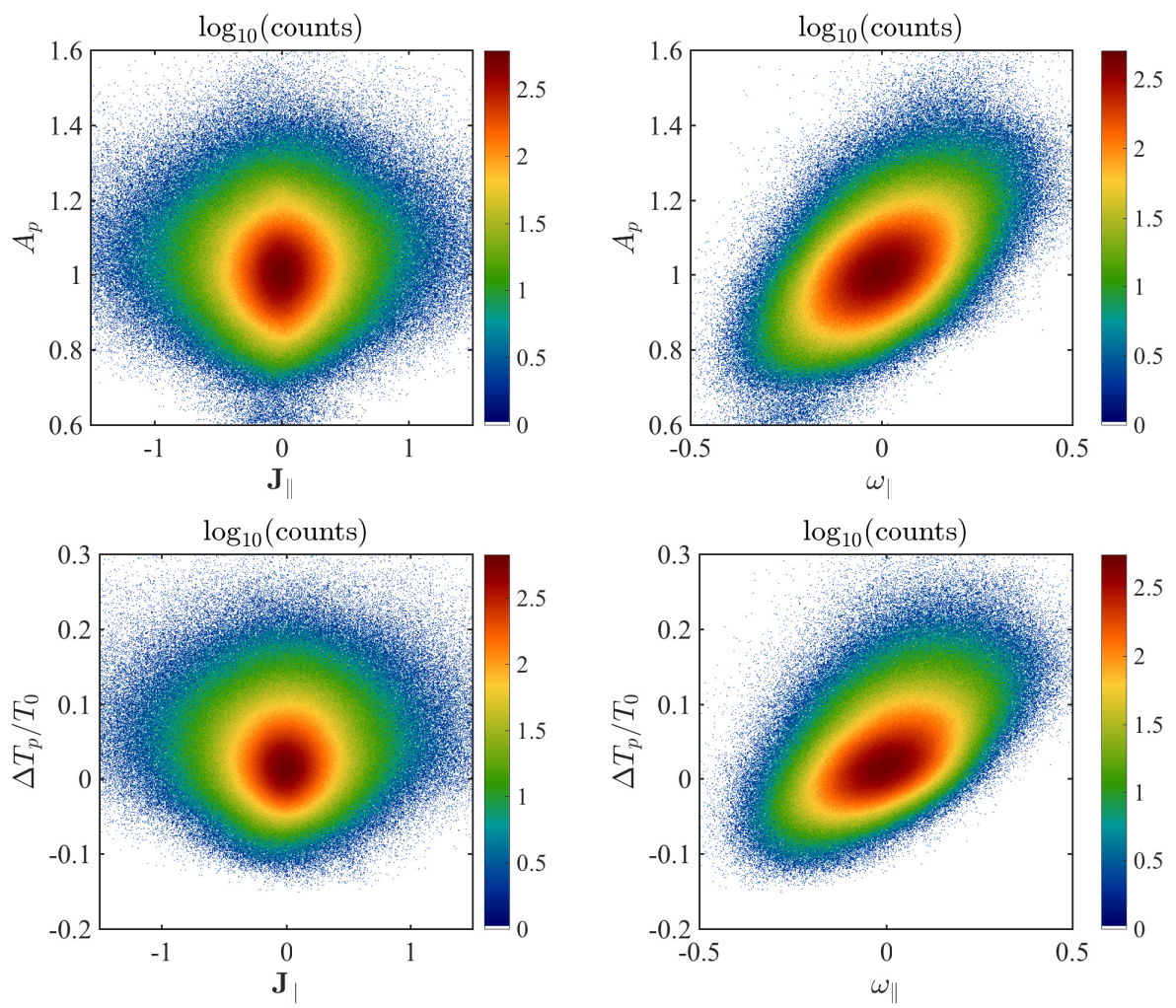

FIGURE 2. Correlations among the four different quantities shown in Fig. 1, i.e., the out-of-plane current density, $\boldsymbol{J}_{\|}$, the out-ofplane vorticity, $\omega_{\|}$, the relative proton temperature variation, $\Delta T_{p} / T_{0}$, and the proton temperature anisotropy, $A_{p}$.

In order to go beyond a qualitative estimate of the correlation among the four above-mentioned quantities, in Fig. 2 we show four scatter plots. In particular, in the top-left and in the bottom-left panels we report the values of the proton temperature anisotropy, $A_{p}$, and of the relative proton temperature variation, $\Delta T_{p} / T_{0}$, respectively, as a function of the out-of-plane current density, $\boldsymbol{J}_{\|}$. No clear correlation with $\boldsymbol{J}_{\|}$can be appreciated for both quantities. In the top-right and in the bottom-right panels we show the value of $A_{p}$ and $\Delta T_{p} / T_{0}$ as a function of the out-of-plane 
vorticity, $\omega_{\|}$. In this case, a correlation with $\omega_{\|}$is clearly present for both quantities, confirming what we have already inferred when looking at the respective isocontour plots.

\section{DISCUSSION}

We have shown that regions with a large proton temperature anisotropy and with a global proton heating seem to be more directly correlated with the structures in the vorticity, rather than with the current density. The local spatial distribution of the latter exhibits a similar pattern, but it has a more complex and filamentary structure, as expected from the spectra of the fluctuations (see $[8,9]$ ), since the magnetic field has much more power than the proton bulk velocity at small scales. A possible explanation for the direct link between the vorticity and the proton temperature could be related to the development of non-gyrotropic ion distribution functions. The presence of such non-gyrotropic distributions has been observed in hybrid-Vlasov simulations [6]. Recently, it has been shown that a sheared velocity field can provide an effective mechanism that makes an initial isotropic state anisotropic and non-gyrotropic [14]. The equations for the evolution of the diagonal terms of the pressure tensor involve the cross-derivatives of the flow velocity components. This could determine the observed correlation between the evolution of the proton temperature and the vorticity. In particular, according to Eq. (2) of [14], the evolution of the 2nd order anisotropic ion moment is related to the sign of the scalar product $\mathbf{B} \cdot \omega$ and therefore, in our case, to the sign of the vorticity alone, since the global magnetic field is always directed along the positive $z$ direction. This is consistent with what we observe, since positive and negative vorticity (and not only its intensity) differently controls the variation in the proton temperatures. In particular, the vorticity is found to be correlated/anticorrelated with the perpendicular/parallel proton temperature, since regions with the positive vorticity exhibit an increase in $T_{p \perp}$ and a decrease in $T_{p \|}$, while the opposite occurs for the negative vorticity. This explains the increase of both the total proton temperature and the proton temperature anisotropy in regions with the positive vorticity. The observed correlations are likely related to a more general relationship between the velocity stress tensor and the pressure tensor. Further work is therefore needed to better clarify the effects of the former (and in particular of the vorticity) on the latter.

\section{ACKNOWLEDGMENTS}

The authors wish to acknowledge valuable discussions with Daniele Del Sarto. This project has received funding from the European Union's Seventh Framework Programme for research, technological development and demonstration under grant agreement no 284515 (SHOCK). Website: http://project-shock.eu/home/. This research was conducted with high performance computing (HPC) resources provided by CINECA (grant HP10CVCUF1). L.M. was funded by STFC grant ST/K001051/1.

\section{REFERENCES}

[1] O. Alexandrova, C. H. K. Chen, L. Sorriso-Valvo, T. S. Horbury, and S. D. Bale 178, 101 (2013).

[2] E. Marsch, R. Schwenn, H. Rosenbauer, K.-H. Muehlhaeuser, W. Pilipp, and F. M. Neubauer, J. Geophys. Res. 87, 52 (1982).

[3] P. Hellinger, P. Trávníček, J. C. Kasper, and A. J. Lazarus 33, L09101 (2006).

[4] A. Greco, F. Valentini, S. Servidio, and W. H. Matthaeus, Phys. Rev. E 86, 066405 (2012).

[5] S. Servidio, F. Valentini, F. Califano, and P. Veltri 108, 045001 (2012).

[6] F. Valentini, S. Servidio, D. Perrone, F. Califano, W. H. Matthaeus, and P. Veltri, Phys. Plasmas 21, 082307 (2014).

[7] S. Servidio, F. Valentini, D. Perrone, A. Greco, F. Califano, W. H. Matthaeus, and P. Veltri 81, 325810107 (2015).

[8] L. Franci, A. Verdini, L. Matteini, S. Landi, and P. Hellinger, ApJ Lett. 804, L39 (2015).

[9] L. Franci, S. Landi, L. Matteini, A. Verdini, and P. Hellinger, ApJ 812, 21 (2015).

[10] C. H. K. Chen, S. Boldyrev, Q. Xia, and J. C. Perez, Phys. Rev. Lett. 110, 225002 (2013).

[11] F. Sahraoui, M. L. Goldstein, G. Belmont, P. Canu, and L. Rezeau, Phys. Rev. Lett. 105, 131101 (2010).

[12] K. H. Kiyani, S. C. Chapman, F. Sahraoui, B. Hnat, O. Fauvarque, and Y. V. Khotyaintsev , ApJ 763, 10 (2013).

[13] S. Servidio, K. T. Osman, F. Valentini, D. Perrone, F. Califano, S. Chapman, W. H. Matthaeus, and P. Veltri, ApJ Lett. 781, L27 (2014).

[14] D. Del Sarto, F. Pegoraro, and F. Califano, ArXiv e-prints (2015), 1507.04895. 